\title{
A GENERALIZED SHILOV BOUNDARY AND ANALYTIC STRUCTURE ${ }^{1}$

\author{
RICHARD F . BASENER
}

\begin{abstract}
A generalization of the concept of the Shilov boundary of a uniform algebra is introduced. This makes it possible to formulate and prove several-dimensional analogues of certain well-known results which guarantee the existence of one-dimensional analytic structure when a function in the algebra is finite-to-one over a suitable part of its spectrum.
\end{abstract}

A major problem in the study of uniform algebras is to find interesting sufficient conditions for the existence of analytic structure in the ir maximal ideal spaces; see, e.g., [6, Chapter 3 and $\$ 30]$. With one notable exception (a result due to Gleason [3], or see [6, Theorem 15.2]), most of these results are one dimensional. In this paper we indicate how one kind of condition ([7, Theorems 10.7 and 11.2], which were derived from Bishop's paper [2]) can be generalized to yield several-dimensional analytic structure. First we must define a generalization of the Shilov boundary of a uniform algebra.

Notation. A will always denote a uniform algebra defined on the compact Hausdorff space $X$ with maximal ideal space $M . \partial_{0} A$ is the usual Shilov boundary of $A$. Let

$$
A^{n}=\left\{\left(f_{1}, \cdots, f_{n}\right) \mid f_{1}, \cdots, f_{n} \in A\right\},
$$

so that each $F=\left(f_{1}, \cdots, f_{n}\right) \in A^{n}$ maps $M$ to $\mathbf{C}^{n}$, and $F(M)$ is the joint spectrum of $f_{1}, \cdots, f_{n}$. If $K$ is a compact subset of $M$, let

$$
A_{K}=\{f \in C(K) \mid f \text { is a uniform limit on } K \text { of functions from } A\} .
$$

If $F \in A^{n}$, let

$$
V(F)=\{x \in M \mid F(x)=0\}
$$

be the $A$-variety corresponding to $F$ and note that $V(F)$ is $A$-convex, i.e., the maximal ideal space of $A_{V(F)}$ is $V(F)$.

Received by the editors August 15, 1973.

AMS (MOS) subject classifications (1970). Primary 46J10, 46J15.

Key words and phrases. Analyticity, maximal ideal space, Shilov boundary.

1 This research was supported in part by NSF grant GP-30671. 
If $Y$ is a topological space, and if $Z \subseteq Y$, then $\partial_{Y} Z$, or simply $\partial Z$, will denote the topological boundary of $Z$ relative to $Y$. For $z \in \mathrm{C}^{n}$ we let $|z|=\left(\sum_{j=1}^{n}\left|z_{j}\right|^{2}\right)^{1 / 2}$, the usual Euclidean norm of $z$, and then we define

$$
B^{n}=\left\{z \in \mathbf{C}^{n}|| z \mid<1\right\}, \quad S^{n}=\left\{z \in \mathbf{C}^{n}|| z \mid=1\right\} .
$$

Finally, $m_{k}$ will denote $k$-dimensional Lebesgue measure; its domain will always be clear from the context.

Definition. $\partial_{n} A=$ Closure $\left[\bigcup\left\{\partial_{0}\left(A_{V(F)}\right) \mid F \in A^{n}\right\}\right]$.

(At this point the reader may wish to look ahead to the statements of Theorems 1 and 2 to see the direction in which our development will proceed.)

Lemma 1. Let $x \in K \subseteq M, K$ closed. Let $F \in A^{n}$ and suppose that $x \in V(F)$. Then $\forall g \in A_{V(F)}$,

$$
|g(x)| \leq \max \left\{|g(y)| \mid y \in[\partial K \cap V(F)] \cup\left[\partial_{n} A \cap V(F) \cap K\right]\right\} .
$$

Proof. This follows at once from the preceding definition and the usual local maximum modulus principle [7, Theorem 9.3] applied to $A_{V(F)}$.

Corollary 1. Let $K$ be a compact $A$-convex subset of $M \backslash \partial_{n} A$. Then $\partial_{n}\left(A_{K}\right) \subseteq \partial K$.

Proof. Suppose that the Corollary is false, so that $\partial_{n}\left(A_{K}\right) \underline{\not} \partial K$. Then there exists $G=\left(g_{1}, \cdots, g_{n}\right) \in\left(A_{K}\right)^{n}$ such that the interior of $K$ meets the Shilov boundary of $\left(A_{K}\right) V(G)$. Consequently there is an $b \in A$ and a $p \epsilon$ int $(K) \cap V(G)$ such that $|h(p)|>1$, but $|h|<1$ on $\partial K \cap V(G)$. Now choose $\epsilon>0$ such that $|h|<1$ on $\left\{x \in \partial K|| g_{j}(x) \mid<\epsilon, 1 \leq j \leq n\right\}$. Let $f_{1}, \cdots, f_{n} \in A$ be chosen so that $f_{j}(p)=0$, while $\left|f_{j}-g_{j}\right|<\epsilon$ on $K, 1 \leq j \leq n$. Then $|b|<1$ on $\partial K \cap V\left(f_{1}, \cdots, f_{n}\right), p \in V\left(f_{1}, \cdots, f_{n}\right)$, and $|b(p)|>1$. This contradicts the local maximum modulus principle of Lemma 1, so the Corollary is established.

Lemma 2. (Cf. [7, Lemma 11.1].) Let $n>0$ and suppose that $\partial_{n-1} A \subseteq X$. Let $F \in A^{n}$ and let $W$ be a component of $\mathbf{C}^{n} \backslash F(X)$. Then either $F(M) \cap W=$ $\varnothing$ or $F(M) \cap W=W$.

Proof. Suppose $\varnothing \neq F(M) \cap W \neq W$. Then $\exists x \in M$ such that $z^{1}=F(x) \epsilon$ $\partial[F(M)] \cap W$. Choose $z^{0} \in W \backslash F(M)$ such that $\left|z^{0}-z^{1}\right|<\alpha=\min \left\{\left|z-z^{0}\right| \mid z\right.$ $\epsilon F(X)\}$. W $\log , z^{0}=0, z^{1}=(1,0, \cdots, 0)$; so $\alpha>1$.

Let $F=\left(f_{1}, \cdots, f_{n}\right)$. Since $0 \notin F(M), \exists h_{1}, \cdots, h_{n} \in A$ such that $\sum_{j=1}^{n} h_{j} f_{j}=1$ (see [7, Lemma 8.1]). Let $G=\left(f_{2}, \cdots, f_{n}\right) \in A^{n-1}$. Since $x \in V(G)$, we have 


$$
\begin{aligned}
\left|h_{1}(x)\right| & \leq \max _{V(G) \cap \partial_{n-1} A}\left|h_{1}\right|=\max _{V(G) \cap X}\left|h_{1}\right| \\
& =\max _{V(G) \cap X} \frac{1}{\left|f_{1}\right|}=\max _{V(G) \cap X} \frac{1}{|F|} \leq 1 / \min _{X}|F|=\frac{1}{\alpha}<1 .
\end{aligned}
$$

But also

$$
h_{1}(x)=\frac{1-\sum_{j=2}^{n} h_{j}(x) f_{j}(x)}{f_{1}(x)}=1 .
$$

This contradiction shows that we must originally have had $F(M) \cap W=\varnothing$ or $F(M) \cap W=W$.

Theorem 1. Let $n>0$ and let $\partial_{n-1} A \subseteq X$. Let $F \in A^{n}$ and suppose that $|F|=1$ on $X$, that $0 \in F(M)$, and that $\exists S^{\prime} \subseteq S^{n}$ such that $m_{2 n-1}\left(S^{\prime}\right)>0$ and $\forall \lambda \in S^{\prime} \exists$ a unique $q \in X$ with $F(q)=\lambda$. Then

$\forall \zeta \in B^{n} \exists$ a unique $x \in M$ with $F(x)=\zeta$;

$\forall f \in A, f \circ F^{-1}$ is holomorphic on $B^{n}$.

Proof. The case $n=1$ is Theorem 10.7 in [7], so we assume that $n>1$. Let $z \in B^{n}$. Since $m_{2 n-1}\left(S^{\prime}\right)>0$, it follows that for some complex line $L=$

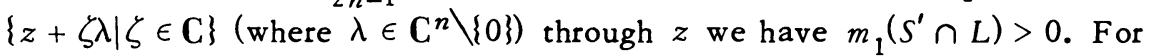
simplicity assume that $\lambda=(1,0, \cdots, 0)$ and that $z=0$, and let $G=$ $\left(f_{2}, \cdots, f_{n}\right)$. One readily verifies that $\left.f_{1}\right|_{V(G)}$ is a function in the uniform algebra $A_{V(G)}$ to which the $n=1$ result can be applied. (Note that since $0 \in F(M)$, Lemma 2 implies that $F(M) \supseteq B^{n}$, so that we are justified in assuming that $z=0$.) We can thus conclude that $\forall z^{\prime} \in B^{n} \cap L, \exists$ precisely one $x^{\prime} \in V(G)$ with $F\left(x^{\prime}\right)=z^{\prime}$. Thus $\forall z \in B^{n}, \exists$ a unique $x \in M$ with $F(x)=z$.

Fix $\rho, 0<\rho<1$. Let

$$
K=\{x \in M|| F(x) \mid \leq \rho\} .
$$

By Corollary 1, $\partial_{n-1}\left(A_{K}\right) \subseteq \partial K=\{x \in M|| F(x) \mid=\rho\}$. Since $F$ is one-to-one on $K$, the result for $n=1$ implies that $\forall f \in A, f \circ F^{-1}$ is holomorphic on the intersection of any complex line with $\left\{z \in \mathbf{C}^{n}|| z \mid<\rho\right\}$. Since $\rho$ was arbitrary, it follows that $\forall f \in A, f \circ F^{-1}$ is holomorphic on $B^{n}$.

Lemma 3. (Cf. [1, Lemma 3].) Let $n>0$ and let $\partial_{n-1} A \subseteq X$. Let $F \in A^{n}$, let $W$ be a component of $\mathrm{C}^{n} \backslash F(X)$, let $z \in W$, and suppose that $J$ is a component of $F^{-1}(z)$. Then for each neighborhood $\Theta$ of J, $F(\Theta)$ is a neighborhood of $z$; given such an $\mathcal{O}, \exists$ a compact $A$-convex neighborbood $N$ of $J$ such that $N \subseteq \mathcal{O}$ and $z \notin F(\partial N)$. 
Proof. Choose compact sets $J^{\prime}, J^{\prime \prime}$ such that $F^{-1}(z)=J^{\prime} \cup J^{\prime \prime} ; J^{\prime} \cap J^{\prime \prime}$ $=\varnothing$, and $J \subseteq J^{\prime} \subseteq \mathcal{O}$ (cf. [6, Lemma 8.13]). $K=F^{-1}(z)$ is $A$-convex, so $\exists g$ $\epsilon A_{K}$ such that $g=0$ on $J^{\prime}, g=1$ on $J^{\prime \prime}$ (by the Shilov idempotent theorem [7, Theorem 8.6]). Choose $h \in A$ with $\max _{K}|h-g|<1 / 4$ and define

$$
U=\{x \in \mathcal{O}|| h(x) \mid<1 / 4\} \cup\{x \in M|| b(x) \mid>3 / 4\}
$$

so that $U$ is a neighborhood of $F^{-1}(z)$. Choose $\epsilon>0$ such that $\{x \in M|| F(x)$ $-z \mid \leq \epsilon\} \subseteq U$. Let

$$
N=\{x \in M|| F(x)-z|\leq \epsilon,| h(x) \mid \leq 1 / 4\} .
$$

Then $N$ is a compact $A$-convex neighborhood of $J$ and $N \subseteq \mathcal{O}$. If $x \in \partial N$, either $|F(x)-z|=\epsilon$ or $|b(x)|=1 / 4$, so that $z \notin F(\partial N)$. Finally, $N \subseteq M \backslash \partial_{n-1} A$, so Corollary 1 implies that $\partial_{n-1}\left(A_{N}\right) \subseteq \partial N$. Since $z \in F(N) \backslash F(\partial N)$, Lemma 2 implies that $F(N)$ is a neighborhood of $z$.

Theorem 2. Let $n>0$ and let $\partial_{n-1} A \subseteq X$. Let $F \in A^{n}$ and let $W$ be a component of $\mathbf{C}^{n} \backslash F(X)$. Assume that $F(M) \cap W \neq \varnothing$. Suppose $\exists W^{\prime} \subseteq W$ such that $m_{2 n}\left(W^{\prime}\right)>0$ and $\forall z \in W^{\prime}$,

$$
\# F^{-1}(z)=(\text { number of } x \in M \text { with } F(x)=z)
$$

is finite. For $l=1,2, \cdots$, let

$$
W_{l}=\left\{z \in W \mid \# F^{-1}(z)=l\right\} .
$$

Then $\exists$ a positive integer $k$ such that

(i) $W=\bigcup_{j=1}^{k} W_{j}$;

(ii) $\bigcup_{j=1}^{k-1} W_{j}$ is a proper analytic subvariety of $W$;

(iii) $F: F^{-1}(W) \rightarrow W$ is a (branched) analytic cover (in the sense of $[5, p .101])$.

Consequently, $\exists$ a sheaf $\mathcal{O}$ of germs of functions on $F^{-1}(W)$ such that $\left(F^{-1}(W), \Theta\right)$ is an analytic space of pure dimension $n$ and $\forall f \in A, f$ is holomorphic on $F^{-1}(W)$ (for definitions, see [5, pp. 147-155]).

Proof. We shall assume that $n>1$, as the $n=1$ case is essentially Theorem 11.2 in [7]. (If $X$ is not metric, any difficulty with the measurability of sets needed in the proof of Theorem 11.2 can be dealt with by the kind of idea developed in Assertion 1 below.) For $l=1,2, \ldots$, let $V_{l}=\bigcup_{j=1}^{l} w_{j}$.

Assertion 1. If $T$ is a relatively closed subset of $W$ and if $\forall z \in T$, $\# F^{-1}(z)$ is finite, then $\forall l, V_{l} \cap T$ is relatively closed and hence $W_{l} \cap T$ is measurable. 
Proof of Assertion 1. Suppose $z \in T, z_{n} \in V_{l} \cap T, z_{n} \rightarrow z$. Let $m=$ $\# F^{-1}(z)<\infty$. By Lemma $3 \exists$ disjoint compact sets $N_{1}, \cdots, N_{m}$ such that $F\left(N_{j}\right)$ is a neighborhood of $z$ for $j=1, \cdots, m$. Thus for $n$ large, $m \leq$ $\# F^{-1}\left(z_{n}\right) \leq l$, so $z \in V_{l}$.

Now let

$$
\begin{aligned}
B(z ; \delta) & =\left\{\zeta \in \mathbf{C}^{n}|| z-\zeta \mid<\delta\right\}, \quad z \in \mathbf{C}^{n}, \delta>0 ; \\
Z_{l} & =\left\{z \in W \mid \forall \delta>0 \exists K \subseteq B(z ; \delta) \cap V_{l} \text { with } K\right. \text { measurable } \\
& \text { and } \left.m_{2 n}(K)>0\right\}, \quad l>0 .
\end{aligned}
$$

(It is clear that the set $Z_{l}$ is closed relative to $W$. Thus the next assertion shows that either $Z_{l}=\varnothing$ or $Z_{l}=W$. )

Assertion 2. $\forall l>0, Z_{l}$ is open and $Z_{l} \subseteq V_{l}$.

Proof of Assertion 2. Let $z^{0} \in Z_{l}$. Choose $\delta>0$ such that $B\left(z^{0} ; \delta\right)$ $\subseteq W$, and let $K$ be a closed subset of $B\left(z^{0} ; \delta\right) \cap V_{l}$ such that $m_{2 n}(K)>0$. Assertion 1 implies that $K \cap W_{j}$ is measurable for each $j$, so $\exists k, 1 \leq k \leq l$, such that $m_{2 n}\left(W_{k} \cap K\right)>0$.

Fix $z \in B\left(z^{0} ; \delta\right)$. It is not hard to show that $\exists \lambda \in \mathbf{C}^{n} \backslash\{0\}$ such that if $L=\{z+\zeta \lambda \mid \zeta \in \mathbf{C}\}$, then $m_{2}\left(L \cap W_{k} \cap K\right)>0$. For simplicity let $z=0, \lambda=$ $(1,0, \cdots, 0)$. Let $G=\left(f_{2}, \cdots, f_{n}\right)$ and define:

$$
\begin{aligned}
& \widetilde{W}=\text { component of } \mathbf{C} \backslash f_{1}(X \cap V(G)) \text { which contains } 0 ; \\
& \widetilde{K}=\left\{\zeta \in \mathbf{C} \mid(\zeta, 0, \ldots, 0) \in W_{k} \cap K\right\} .
\end{aligned}
$$

Then $\left.f_{1}\right|_{V(G)} \in A_{V(G)}$ and is $k$-to-1 over $\widetilde{K}$, so the one-dimensional version of Theorem 2 shows that $\forall \lambda \in \widetilde{W}, \#\left[\left(f_{1}\right)^{-1}(\lambda)\right] \leq k$. (Compare Assertion 3 in the proof of Theorem 11.2 in [7]; the " $k$ " there is the same as our " $k$ " as may be verified from its definition on p. 65.) Thus $\# F^{-1}(0) \leq k$; so $\forall z \epsilon$ $B\left(z^{0} ; \delta\right), \# F^{-1}(z) \leq k$, or $B\left(z^{0} ; \delta\right) \subseteq V_{k}$, so $B\left(z^{0} ; \delta\right) \subseteq Z_{l}$. This concludes the proof of Assertion 2.

We are ready to prove (i). Wlog $W^{\prime}$ is closed. Since $W^{\prime}=\bigcup_{l=1}^{\infty} W^{\prime} \cap W_{l}$, Assertion 1 implies that each $W^{\prime} \cap W_{l}$ is measurable, so there is a positive integer $k$ such that $m_{2 n}\left(W^{\prime} \cap W_{k}\right)>0$. Then $Z_{k} \neq \varnothing$, so the connectedness of $W$ and Assertion 2 imply that $Z_{k}=W$; thus $W \subseteq V_{k}=\bigcup_{l=1}^{k} W_{l}$. Note also that if $l<k$, the same argument implies that we must have $m_{2 n}\left(W_{l}\right)=0$.

Assertion 3. If $z^{0} \in W_{k}, \exists \delta>0$ with $B\left(z^{0} ; \delta\right) \subseteq W_{k}$ such that for each component $N$ of $F^{-1}\left(B\left(z^{0} ; \delta\right)\right)$ we have:

$$
\begin{aligned}
& \left.F\right|_{N}: N \rightarrow B\left(z^{0} ; \delta\right) \text { is a homeomorphism; } \\
& \forall g \in A, g \circ\left(\left.F\right|_{N}\right)^{-1} \text { is holomorphic on } B\left(z^{0} ; \delta\right) .
\end{aligned}
$$


Thus it follows that $F: F^{-1}\left(W_{k}\right) \rightarrow W_{k}$ is a k-sheeted covering map.

Proof of Assertion 3. Fix $z^{0} \in W_{k}$, and let $F^{-1}\left(z^{0}\right)=\left\{x_{1}, \cdots, x_{k}\right\}$. Let $N_{1}, \cdots, N_{k}$ be disjoint closed neighborhoods of $x_{1}, \cdots, x_{k}$. Lemma $3 \mathrm{im}$ plies that $F\left(N_{j}\right)$ is a neighborhood of $z^{0}$ for each $j$. Choose $\delta>0$ such that

$$
\bar{B}\left(z^{0} ; \delta\right)=\left\{z \in \mathbf{C}^{n}|| z-z^{0} \mid \leq \delta\right\} \subseteq \mathbb{W} \cap\left(\bigcap_{j=1}^{k} F\left(N_{j}\right)\right) .
$$

Since $W=V_{k}$, we have that for each $z \in \bar{B}\left(z^{0} ; \delta\right)$ and for each $j, \exists$ a unique $x \in N_{j}$ with $F(x)=z$. Let $M_{j}=N_{j} \cap F^{-1}\left(\bar{B}\left(z^{0} ; \delta\right)\right), 1 \leq j \leq k$. Since $M_{j}$ is compact and $F$ maps $M_{j}$ one-to-one onto $\bar{B}\left(z^{0} ; \delta\right)$, the restriction of $F$ to $M_{j}$ is a homeomorphism onto $\bar{B}\left(z^{0} ; \delta\right)$. Let $X_{j}=\left\{x \in M_{j}|| F(x)-z^{0} \mid=\delta\right\}=\partial M_{j}$. Corollary 1 implies that $\partial_{n-1} A_{M_{j}} \subseteq X_{j}$. Fix $j$ and let $N=M_{j} \backslash X_{j}$. Theorem 1 now shows that $\forall g \in A, g \circ\left(\left.F\right|_{N}\right)^{-1}$ is holomorphic on $\bar{B}\left(z^{0} ; \delta\right)$. This completes the proof of Assertion 3.

To prove (ii), define $p_{j}: W_{k} \rightarrow M, 1 \leq j \leq k$, by requiring that $F^{-1}(z)=$ $\left\{p_{1}(z), \cdots, p_{k}(z)\right\}, z \in W_{k}$, the $p_{j}$ being otherwise arbitrary. For each $f \in A$, define

$$
\Delta[f](z)=\left\{\begin{array}{l}
\prod_{i \neq j}\left(f\left(p_{i}(z)\right)-f\left(p_{j}(z)\right)\right), \quad z \in \mathbb{W}_{k} \\
0, \quad z \in \mathbb{W} \backslash \mathbb{W}_{k^{*}}
\end{array}\right.
$$

Assertion 3 shows that $\forall f \in A, \Delta[f]$ is holomorphic on the open set $W_{k}$, and the remark preceding Assertion 3 shows that $W_{k}$ is dense in $W$. If $z^{0} \epsilon$ $W \backslash W_{k}$, then $\# F^{-1}\left(z^{0}\right)<k$ and Lemma 3 implies that for each $f \in A$,

$$
\lim _{z \rightarrow z^{0} ; z \in W_{k}} \Delta[f](z)=0 .
$$

Thus we may apply Rado's theorem (the $n=1$ case is Theorem 10.6 in [7]) to conclude that $\forall f \in A, \Delta[f]$ is holomorphic on $W$. Since the functions in $A$ separate points on $M$,

$$
\bigcup_{j=1}^{k-1} W_{j}=\mathbb{W} \backslash W_{k}=\bigcap_{f \in A}\{z \in \mathbb{W} \mid \Delta[f](z)=0\} .
$$

Thus $\bigcup_{j=1}^{k-1} W_{j}$ is a proper analytic subvariety of $W$.

Now we have almost all the information needed to conclude that $F: F^{-1}(W) \rightarrow W$ is a $k$-sheeted (branched) analytic covering of $W$ in the sense of $[5, \mathrm{p} .101]$. In fact, the only conditions in this definition of analytic 
cover which we have not already verified are that the restriction of $F$ to $F^{-1}(W)$ is a proper map and that $F^{-1}\left(W \backslash \bigcup_{j=1}^{k-1} W_{j}\right)$ is dense in $F^{-1}(W)$. The first condition is an immediate consequence of the fact that $F$ is defined and continuous on all of the compact set $M$, and the second condition follows from an application of Lemma 3 as in Assertion 3.

The fact that an analytic cover is an analytic space may be found in [4, Theorem 32]. If $f \in A$, Assertion 3 and Definition 4 in [5, p. 101] now imply that the restriction of $f$ to $F^{-1}(W)$ is holomorphic.

Theorem 3. Let $n>0$ and let $\partial_{n-1} A \subseteq X$. Let $F \in A^{n}$ and let $W$ be $a$ component of $\mathbf{C}^{n} \backslash F(X)$. Assume that $\forall z \in W, F^{-1}(z)$ is at most countable. Then $\exists$ an open dense subset $U$ of $F^{-1}(W)$ and a sheaf $\mathcal{O}$ of germs of functions on $U$ such that $(U ; O)$ is an $n$-dimensional complex analytic manifold and $\forall f \in A, f$ is holomorphic on $U$.

Proof. The proof is virtually identical with that of the Theorem in [1], so we shall not repeat it here. (Simply substitute Theorem 1, Lemma 2 and Lemma 3 above for Lemmas 1, 2 and 3 in the proof of the Theorem in [1].)

\section{REFERENCES}

1. R. F. Basener, A condition for analytic. structure, Proc. Amer. Math. Soc. 36 (1972), 156-160. MR $46 \# 7903$.

2. E. Bishop, Holomorphic completions, analytic continuation, and the interpolation of semi-norms, Ann. of Math. (2) 78 (1963), 468-500. MR 27 \#4958.

3. A. M. Gleason, Finitely generated ideals in Banach algebras, J. Math. Mech. 13 (1964), 125-132. MR 28 \#2458.

4. H. Grauert and R. Remmert, Komplexe Räume, Math. Ann. 136 (1958), 245318. MR $21 \# 2063$.

5. R. C. Gunning and H. Rossi, Analytic functions of several complex variables, Prentice-Hall Series in Modern Analysis, Prentice-Hall, Englewood Cliffs, N. J., 1965. MR $31 \# 4927$.

6. E. L. Stout, The theory of uniform algebras, Bogden \& Quigley, Tarrytownon-Hudson, N. Y., 1971.

7. J. Wermer, Banach algebras and several complex variables, Markham, Chicago, Ill., 1971. MR 46 \#672.

DEPARTMENT OF MATHEMATICS, YALE UNIVERSITY, NEW HAVEN, CONNECTICUT 06520

Current address: Department of Mathematics, Lehigh University, Bethlehem, Pennsylvania 18015 